\title{
Investigating the hyperon-nucleon strong interaction with a precision measurement of the hypertriton lifetime in ALICE
}

\author{
Francesco Mazzaschi*, on behalf of the ALICE collaboration \\ Università and INFN Torino \\ E-mail: francesco.mazzaschi@cern.ch
}

\begin{abstract}
The hypertriton is a bound state of a proton, a neutron and a $\Lambda$ baryon. Studying its internal structure is crucial to investigate the hyperon-nucleon strong interaction and offers insights into the inner core of neutron stars, where $\Lambda$ production is favoured. One powerful way to investigate the hypertriton's internal structure is to measure precisely its lifetime. The hypertriton is an extremely loosely bound object with a lambda separation energy $E_{\Lambda}$ of only $130 \mathrm{keV}$. Therefore the hypertriton lifetime is expected to be close to the one of the free $\Lambda$. Thanks to the very large set of $\mathrm{Pb}-\mathrm{Pb}$ collision data collected during LHC Run 2, the ALICE collaboration has performed systematic studies on the hypertriton lifetime. The hypertriton 2-body mesonic decay channel has been studied with the help of machine learning techniques for the signal extraction. The precision of the new ALICE results on the hypertriton lifetime is comparable with the current world average and the measured lifetime value confirms the weakly-bound nature of the hypertriton.
\end{abstract}

$40^{\text {th }}$ International Conference on High Energy Physics

Prague, Czech Republic

28/07/2020 - 06/08/2020

\footnotetext{
${ }^{*}$ Speaker
} 


\section{Physics motivations}

The hypertriton $\left({ }_{\Lambda}^{3} \mathrm{H}\right)$ is the lightest known hypernucleus, being composed of a proton, a neutron and a $\Lambda$ baryon. Studying its properties gives information on the hyperon-nucleon (Y-N) interaction. The Y-N interaction study has become relevant in recent years because many theoretical models predict the production of $\Lambda$ hyperons in the inner core of the neutron stars, leading to a softening of the matter equation of state [1]. As a consequence, the formation of large mass neutron stars, such as those observed [2], should be suppressed. In the literature this incongruence is often referred to "the hyperon puzzle". One of the most promising classes of models which can cope with this problem is based on the introduction of three-body forces. Indeed, the introduction of an additional repulsion term in the interaction potential can counterbalance the large gravitational pressure, allowing for the formation of stars of larger masses [3]. A detailed knowledge of the Y-N interaction and of the three-body Y-N-N interaction is fundamental for testing the robustness of these models. Since the measurement of the hypertriton lifetime gives information on its internal structure, a precise measurement is crucial for understanding the $\mathrm{Y}-\mathrm{N}$ interaction. Moreover, the $\Lambda$ separation energy $\left(\mathrm{E}_{\Lambda}\right)$ in this hypernucleus is only $130 \mathrm{keV}$ [4]. Such a low $\mathrm{E}_{\Lambda}$ implies a small change of the $\Lambda$ wave function inside the nucleus, hence the hypertriton lifetime is expected to be close to the free $\Lambda$ hyperon. Accurate calculations from Kamada et al. [5] estimated a value of $\tau\left({ }_{\Lambda}^{3} \mathrm{H}\right)=256 \mathrm{ps}$ for ${ }_{\Lambda}^{3} \mathrm{H}$, to be compared with the Particle Data Group (PDG) value for the free $\Lambda: \tau(\Lambda)=(263.2 \pm 2.0)$ ps [6].

Since its discovery in the 60 s to the early 90 s, the hypertriton used to be detected using imaging techniques (i.e. emulsions, bubble chambers) [4]. Many of these measurements were carried out using small data samples, resulting in large statistical uncertainties, thus preventing conclusive statements on the existing model calculations. Recent measurements of the lifetime were performed in ultrarelativistic heavy-ion collisions and in relativistic ion fragmentation experiments. The hypertriton lifetime measured in Au-Au collisions by the STAR Collaboration at RHIC [7] triggered a particular interest for a more precise determination of the ${ }_{\Lambda}^{3} \mathrm{H}$ lifetime. The STAR result was followed by two measurements. The first one was carried out by the HypHI Collaboration at GSI and was based on the projectile fragmentation of ${ }^{6} \mathrm{Li}$ at $2 \mathrm{GeV}$ on a carbon target [8]. The other one was performed by ALICE in $\mathrm{Pb}-\mathrm{Pb}$ collisions at $\sqrt{\mathrm{s}_{\mathrm{NN}}}=2.76 \mathrm{TeV}$ [9]. All three measurements, even though affected by statistical and systematic uncertainties larger than $10 \%$, are in agreement and are lower than the free $\Lambda$ lifetime. In particular, the STAR measurement is about $50 \%$ lower than the $\Lambda$ lifetime. The ALICE Collaboration has measured the lifetime with better precision using data collected in $\mathrm{Pb}-\mathrm{Pb}$ during the 2015 campaign [10]. This measurement is consistent within $1 \sigma$ with both the world average (computed including all the previous measurements) and the $\Lambda$ lifetime. The difficulty to reconcile the experimental values previously reported and the small binding energy of the system represents the so called "lifetime puzzle" that the new ALICE measurement aims to solve.

\section{Analysis method}

The measurement presented in this contribution is performed using $\mathrm{Pb}-\mathrm{Pb}$ data collected during 2018, which consists of 250 million events. The (anti-)hypertriton reconstruction is performed 
analysing its two-body mesonic charged decay channel ${ }_{\Lambda}^{3} \mathrm{H} \rightarrow{ }^{3} \mathrm{He}+\pi^{-}$(and the related charge conjugated particles for $\frac{3}{\Lambda} \bar{H}$ ). Pions and (anti- $)^{3} \mathrm{He}$ are identified using the measured energy loss in the Time Projection Chamber (TPC), which has excellent particle identification capabilities. Then a vertexing algorithm performs the secondary vertex reconstruction, in which all the ${ }^{3} \mathrm{He}$ and $\pi$ tracks satisfying loose topological requirements are matched. Finally the (anti-)hypertriton selection is entrusted to a supervised machine learning algorithm that allows for a better separation between the signal and the background. ${ }_{\Lambda}^{3} \mathrm{H}\left(\frac{3}{\Lambda} \overline{\mathrm{H}}\right)$ candidates are selected using a Boosted Decision Trees classifier (BDT) [11] trained on a dedicated Monte Carlo (MC) simulated event sample. The BDT is a supervised learning algorithm that discriminates between two or more classes, signal and background in our case, starting from a set of examples, called the training set. In this analysis the training set is composed of (anti-)hypertriton signal candidates extracted from the MC sample and background candidates from paired like-sign ${ }^{3} \mathrm{He}$ and $\pi$ tracks from data. To evaluate the performance of the models, a fraction of the training sample is excluded from the training process and it is only used as a test sample. The output of the BDT for the signal and the background samples is reported in Fig. 1 for both the training and the test samples showing large score separation between the two distributions.

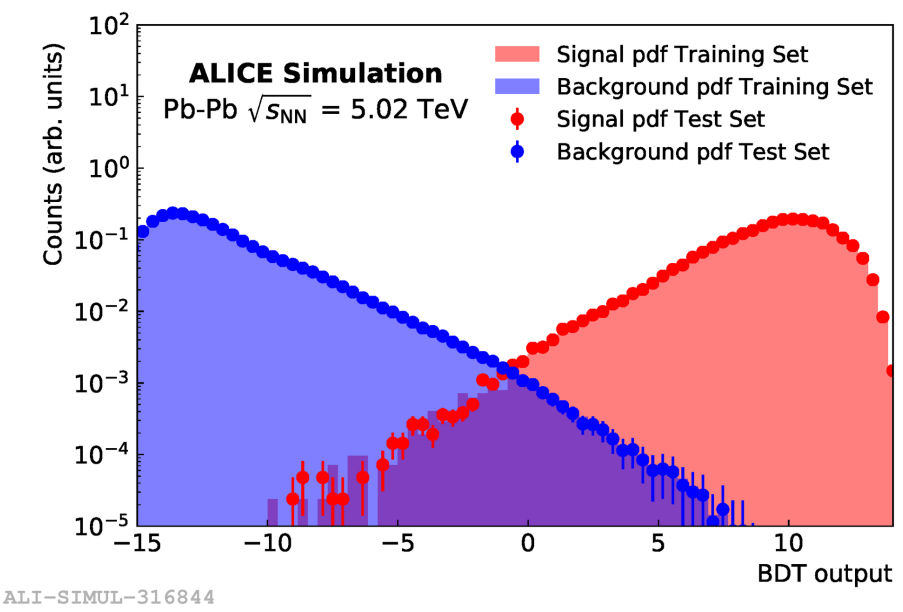

Figure 1: Normalized BDT output distributions for the training (shaded area) and test (points) samples.

The use of the BDT algorithm reduces the number of variables employed for the selection. In fact, instead of a list of selections involving topological and kinematic variables, the ${ }_{\Lambda}^{3} \mathrm{H}\left(\frac{3}{\Lambda} \overline{\mathrm{H}}\right)$ candidates are selected only on the basis of the of BDT score, or threshold. The BDT threshold is chosen by maximising the expected significance, where the expected hypertriton signal is estimated assuming thermal production [12] for ${ }_{\Lambda}^{3} \mathrm{H}\left(\frac{3}{\Lambda} \overline{\mathrm{H}}\right)$ while the expected background is extrapolated from a polynomial fit in the side-band region. The candidates that pass the BDT threshold cut are used to populate the invariant mass spectrum, which is fitted with a Gaussian and a second-order polynomial function to describe the signal and the background component, respectively. The signal is extracted in nine $c \mathrm{t}=\frac{m c}{p} \ell$ intervals, where $c$ is the speed of light, $\mathrm{t}$ is the proper time of the candidate, $m$ is the mass of the candidate, $\ell$ is the decay distance and $\mathrm{p}$ is the reconstructed momentum. The signal extraction in the $2 \leq c \mathrm{t}<4 \mathrm{~cm}$ interval is reported in Fig. 2, as an example. 


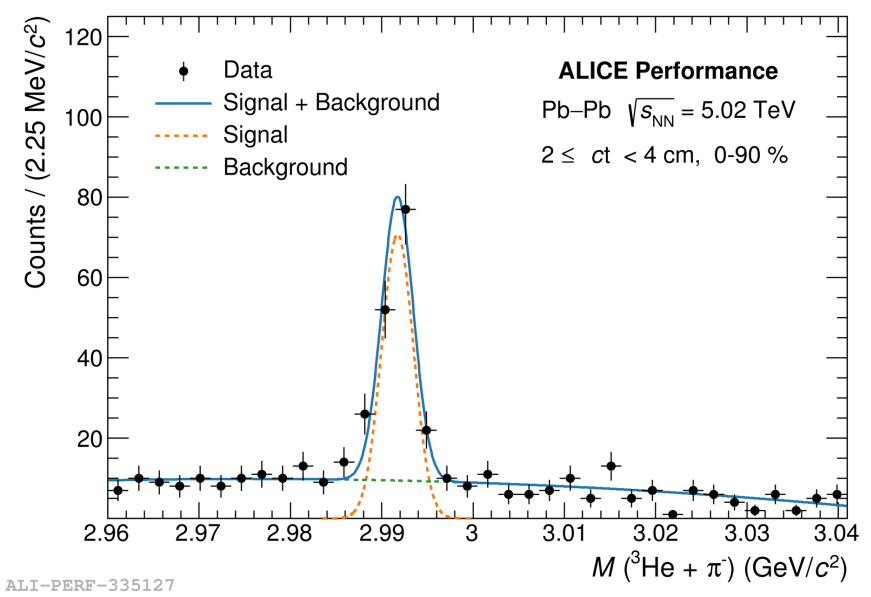

Figure 2: Hypertriton invariant-mass distribution in the proper decay length interval $2 \leq c \mathrm{t}<4 \mathrm{~cm}$ and fit used for the signal extraction.

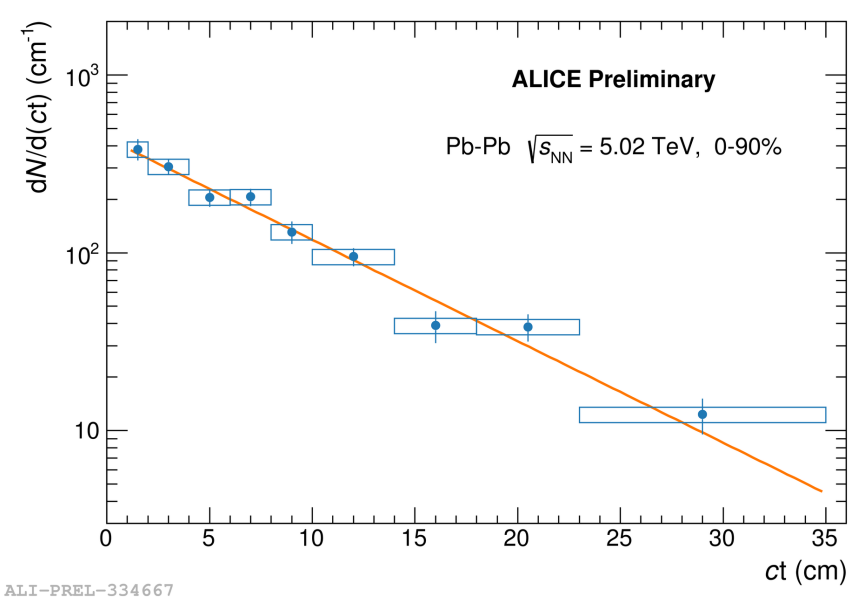

Figure 3: $d N / d(c t)$ spectrum with statistical (bar) and systematic (box) uncertainties. The orange line corresponds to the exponential fit performed to measure the lifetime.

\section{Results}

The signal extracted from the invariant mass spectra in each $c$ interval is corrected for the efficiency and the absorption in the detector material, both estimated by using MC simulations based on GEANT 4 [13]. The corrected $c$ t spectrum is shown in Fig. 3. 
The lifetime value of the ${ }_{\Lambda}^{3} \mathrm{H}\left(\frac{3}{\Lambda} \overline{\mathrm{H}}\right)$ is extracted from an exponential fit to this $c$ t spectrum. The value of the ${ }_{\Lambda}^{3} \mathrm{H}\left(\frac{3}{\Lambda} \overline{\mathrm{H}}\right)$ lifetime that is obtained is:

$$
\tau\left({ }_{\Lambda}^{3} \mathrm{H}\right)=\left(254 \pm 15^{\text {(stat. })} \pm 17^{\text {(syst. })}\right) \text { ps. }
$$

The systematic uncertainties are computed considering the contributions from the BDT selection and signal extraction, the absorption in the detector and the material budget.

\section{Conclusions}

Figure 4 summarises all the hypertriton lifetime measurements carried out in the last 56 years. The measurement of the lifetime presented in this contribution is the most precise ever achieved. It is consistent with the previous measurements performed by ALICE and with the theoretical models that assume a weakly bound system. On the contrary, models assuming a compact hypertriton such as [14] are disfavoured by this measurement.

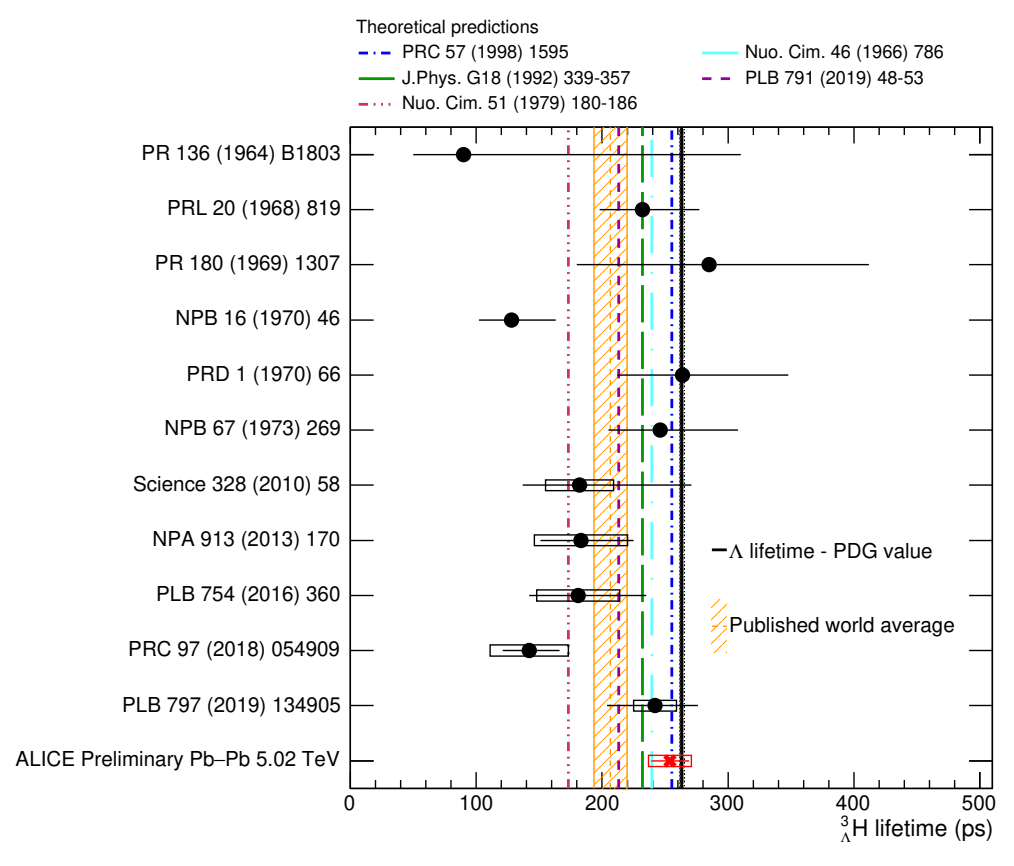

ALI-DER-358857

Figure 4: Collection of the ${ }_{\Lambda}^{3} \mathrm{H}$ and $\frac{3}{\Lambda} \overline{\mathrm{H}}$ lifetime measurements obtained with different experimental techniques. The vertical lines and boxes are the statistical and systematic uncertainties respectively. The orange band represents the average of the lifetime values and the corresponding $1 \sigma$ uncertainty. The dashed-dotted lines are five theoretical predictions. 


\section{References}

[1] L. Tolos, M. Centelles and A. Ramos, The Equation of State for the Nucleonic and Hyperonic Core of Neutron Stars, Publ. Astron. Soc. Austral. 34 (2017) e065 [1708. 08681].

[2] J. Lattimer and M. Prakash, The physics of neutron stars, Science 304 (2004) 536 [astro-ph/0405262].

[3] L. Tolos and L. Fabbietti, Strangeness in Nuclei and Neutron Stars, Prog. Part. Nucl. Phys. 112 (2020) 103770 [2002.09223].

[4] D. Davis, 50 years of hypernuclear physics. I. The early experiments, Nucl. Phys. A 754 (2005) 3.

[5] H. Kamada, J. Golak, K. Miyagawa, H. Witala and W. Gloeckle, Pi mesonic decay of the hypertriton, Phys. Rev. C 57 (1998) 1595 [nucl-th/9709035].

[6] Particle Data Group collaboration, Review of Particle Physics, PTEP 2020 (2020) $083 \mathrm{C} 01$.

[7] STAR collaboration, Measurement of the ${ }_{\Lambda}^{3} H$ lifetime in Au+Au collisions at the BNL Relativistic Heavy Ion Collider, Phys. Rev. C 97 (2018) 054909 [1710. 00436].

[8] C. Rappold et al., Hypernuclear spectroscopy of products from 6Li projectiles on a carbon target at 2 AGeV, Nucl. Phys. A 913 (2013) 170 [1305.4871].

[9] ALICE collaboration, ${ }_{\Lambda}^{3} \mathrm{H}$ and ${ }_{\bar{\Lambda}}^{3} \overline{\mathrm{H}}$ production in Pb-Pb collisions at $\sqrt{s_{\mathrm{NN}}}=2.76 \mathrm{TeV}$, Phys. Lett. B 754 (2016) 360 [1506.08453].

[10] ALICE collaboration, ${ }_{\Lambda}^{3} \mathrm{H}$ and ${ }_{\bar{\Lambda}}^{3} \overline{\mathrm{H}}$ lifetime measurement in Pb-Pb collisions at $\sqrt{s_{\mathrm{NN}}}=5.02$ TeV via two-body decay, Phys. Lett. B 797 (2019) 134905 [1907.06906].

[11] T. Chen and C. Guestrin, XGBoost: A Scalable Tree Boosting System, 1603.02754.

[12] P. Braun-Munzinger and B. Dönigus, Loosely-bound objects produced in nuclear collisions at the LHC, Nucl. Phys. A 987 (2019) 144 [1809. 04681].

[13] GEANT4 collaboration, GEANT4-a simulation toolkit, Nucl. Instrum. Meth. A 506 (2003) 250.

[14] H. Mansour and K. Higgins, The decay rate of hypertriton, Nuovo Cim. A 51 (1979) 180. 